\title{
Signatures of 2-day wave in the E-region electric fields and their relationship to winds and ionospheric currents
}

\author{
H. C. Aveiro, C. M. Denardini, and M. A. Abdu \\ Instituto Nacional de Pesquisas Espaciais, P.O. Box 515, S. J. Campos, SP, Brazil \\ Received: 30 September 2008 - Revised: 17 December 2008 - Accepted: 14 January 2009 - Published: 9 February 2009
}

\begin{abstract}
We analyze the effects of the 2-day wave activity in the EEJ using one coherent scatter radar and eight magnetometer stations located close to the dip equator. The wavelet analysis of the magnetometer data reveals a 2-day signature in the semidiurnal geomagnetic tide. The E-region zonal background ionospheric electric field, derived from coherent radar measurements, shows 2-day oscillations in agreement with such oscillations in the magnetometers data. An anticorrelation between the amplitude of the tidal periodicites (diurnal and semidiurnal) and that of the 2-day signature is also shown in the electric fields. The results are compared with simultaneous observations of 2-day planetary wave in meridional winds and ionosonde data. Further, our results are discussed based on the analysis of the magnetic activity.
\end{abstract}

Keywords. Ionosphere (Equatorial ionosphere)

\section{Introduction}

The equatorial electrojet (EEJ) is an electric current that flows in the height region from about 90 to $120 \mathrm{~km}$ (at ionospheric E-region), covering a latitudinal range of $\pm 3^{\circ}$ centered at the dip equator (Forbes, 1981). It is driven by the E-region dynamo electric field (Fejer and Kelley, 1980) and represents an important aspect of the phenomenology of the equatorial ionosphere-thermosphere system. Sounding observations of the equatorial ionospheric E-region using VHF radars have shown backscattered echoes from electron density irregularities in the EEJ. These echoes contain Doppler shifted frequency components due to the drifts of two types of irregularities, called Type I and Type II. Irregularities in the EEJ related to the Gradient Drift plasma instability are known as Type II. Their Doppler signatures are characterized

Correspondence to: H. C. Aveiro (aveiro@dae.inpe.br) by a broader spectrum with amplitude smaller than that of the Type I irregularities. On magnetically quiet days Type II echoes are founded predominatly in the lower portion of the EEJ. They may be present since early morning hours until sunset. However, the height range where those echoes dominate on magnetically quiet days seems to rise during disturbed periods (Abdu et al., 2003).

Planetary scale equatorial waves play an important role in the tropical atmosphere dynamics. These waves are forced by large-scale unsteady convective clusters in the tropical troposphere and accompanying latent heat release. They propagate horizontally and vertically carrying momentum to the middle and upper atmosphere (Pancheva et al., 2008). Forbes and Leveroni (1992) found a quasi 16-day oscillation in the the equatorial ionosphere likely connected with the upward penetration of a free Rossby mode. Parish et al. (1994) identified 2-, 5-, 10- and 16-day periodicities in the EEJ, which they attributed to planetary waves; however, their analysis suggested also that nonlinear interactions between the planetary waves and the diurnal and semidiurnal tides could be another driving source of these oscillations. Gurubaran et al. (2001) identified the signatures of the quasi-2-day variability in the equatorial electrojet using magnetometers and showed a reasonable correlation with the 2-day wave measured by the MF radar situated close to the geomagnetic stations. Abdu et al. (2006) found planetary wave modulation in the equatorial F-layer vertical drift. In view of the theoretical difficulty of modelling the propagation of such waves to ionospheric heights (Hagan et al., 1993; Forbes, 1995), those results were related to the electrodynamical signature of the interaction between planetary waves and the ionospheric dynamo region. We have many possible scenarios to the explanation of the detection of planetary wave signatures in the ionosphere (Forbes, 1996; Lastovicka, 2006):

- stratospheric/mesospheric planetary waves could modulate the accessibility of gravity waves to the upper

Published by Copernicus Publications on behalf of the European Geosciences Union. 
regions of the atmosphere, involving mechanisms that could lead to secondary sources of excitation of planetary waves in the upper atmosphere;

- planetary wave modulation of vertical plasma drift due to planetary wave modulation of the E-region dynamo; and

- planetary wave modulated turbopause height and turbopause region properties or composition changes at the base of the thermosphere; and

- modulation by planetary waves of the upward propagating tides that participate in the dynamo action to generate electric fields in the upper regions.

The last mechanism seems to be promissing and easier to verify since the forcing by diurnal and semidiurnal tides that propagate upward is basically responsible for the fenomenology of the ionosphere in quiet periods, especially in equatorial latitutes (Abdu et al., 2006).

In the present paper, we analyze the effects of the 2-day wave activity in the EEJ using data from one coherent radar and eight magnetometer stations close to the dip equator (dip lat $<\sim 5^{\circ}$ ). Our results are compared with previous results obtained by Pancheva et al. (2006), who analyzed the 2-day wave coupling of the low-latitude atmosphere-ionosphere system in the neutral winds by radar measurements at four tropical stations, in the ionospheric electron density from seven ionosonde stations, and in the ionospheric electric currents from twenty-three magnetometer stations, during the period between 1 December 2002 and 28 February 2003. Still, our results are discussed based on the analysis of the magnetic activity through the use of $\mathrm{AE}$, and $D_{s t}$ indices.

\section{Two-day wave in the E-region electric fields}

\subsection{Radar experimental description}

The $50 \mathrm{MHz}$ RESCO radar is located at the Brazilian equatorial site, Sao Luiz, at $2.51^{\circ}$ south, $44.27^{\circ}$ west, $-0.5^{\circ}$ dip latitude. The CoCo (coaxial-collinear) antenna array used with the radar consists of 32 strings of 24 dipoles separated by a half wavelength, totalizing 768 dipoles. The array is configured so that the antenna beam can be steered electronically between the vertical and one of the oblique directions ( $\pm 30^{\circ}$ zenith angle) or between the two oblique directions. The theoretical beam width is $\sim 7^{\circ}$ in E-W and $\sim 3^{\circ}$ in N-S planes and the transmitter peak power is $\sim 40 \mathrm{~kW}$.

The radar is usually set for EEJ sounding. The inter-pulse period (IPP) of transmission is usually set to $1 \mathrm{~ms}$. The time delay (TD) between transmission and data acquisition is set to $620 \mu \mathrm{s}$. The observations have been made using a pulse width (PW) of $20 \mu \mathrm{s}$ that corresponds to $3 \mathrm{~km}$ height resolutions using the vertical beam or $2.6 \mathrm{~km}$ when the radar beam is oblique. The radar data acquisition system samples the echoes so that the height coverage is between around 80 and $120 \mathrm{~km}$. The backscattered echo received by the antenna array is amplified before passing through two phase-coherent detectors that provide in-phase and quadrature signals containing the Doppler frequency and power information. The phase detected signals are sampled in 16 range gates and stored in a sequential binary format. The signals are grouped in sets corresponding to 256 pulses (NP) for each sampled range gate. The data processing consisted in an offline spectral analysis using Fast Fourier Transform (FFT) for each range gate of NP data points which resulted in the spectral distribution of the Doppler frequencies contained in the returned signal for each range gate. For the periods of analysis, the time resolution between each set of NP pulses is $12 \mathrm{~s}$ and the aliasing frequency for each spectrum is $500 \mathrm{~Hz}$ with $\sim 4 \mathrm{~Hz}(\mathrm{NP}=256)$ of frequency resolution. Then, by integrating each spectrogram in frequency, we obtained the total power received from each height, and the time variation of the total power in all the range gates is used for plotting the daily Range Time Intensity (RTI) maps.

\subsection{Electric fields inference}

The Doppler spectrum of the echoes is a composite of both Type I and Type II irregularities present inside the volume sampled by the radar. Assuming that the experimental spectra can be decomposed in various Gaussian spectra, the spectral decomposition technique involves fitting the sum of two Gaussian curves to the spectrum. Each Gaussian (related to one specific irregularity type) is characterized by three parameters: center of frequency distribution (corresponding to Doppler shift), spectral power density, and spectral width. After inverting the curves, the six statistical moments (three to each irregularity type) are evaluated. Fitted curves with spectral power smaller than $5 \%$ of the maximum spectral power for the whole day are discarded to avoid eventual bad fitting related-problems. Finally, the phase velocity estimates for Type II irregularities $\left(V_{p}\right)$ which was obtained by fitting, is used to calculate the vertical electric field $\left(E_{z}\right)$ by:

$E_{z}=V_{p}(1+\psi) \cdot \frac{B^{2}}{H}$,

where $H$ is the horizontal component of the magnetic flux density $\mathbf{B}, B$ is defined as $|\mathbf{B}|$, and the anisotropic factor $\psi$ is given by:

$\psi=\frac{v_{i} \cdot v_{e}}{\Omega_{i} \cdot \Omega_{e}}$,

where $\Omega_{e, i}$ is the gyrofrequency and $v_{e, i}$ is the collision frequency. The subscript $e$ and $i$ indicate the electron and ion terms, respectively.

In the computation of $\psi$, we neglected the ion-electron collisions, and the ion-neutral collision frequency were calculated using Kelley (1989):

$v_{i n}=\left(2.6 \times 10^{-9}\right) \cdot\left(N_{n}+N_{i}\right) \cdot\left(A_{n}+A_{i}\right)^{-1 / 2}$, 
where $N_{n, i}$ is the density and $A_{n, i}$ is the average molecular weight, respectivelly. The subscript $n$ indicates the neutral terms. We neglect the ion terms, so $N_{n}+N_{i} \approx N_{n}$ and $A_{n}+A_{i} \approx A_{n}$. Since the electron-ion collisions are negligible in the lower E-region (Forbes, 1981), the electron collision frequency is approximated by the electron-neutral collisions, as given by (Kelley, 1989):

$v_{e n}=\left(5.4 \times 10^{-10}\right) \cdot N_{n} \cdot T_{e}^{1 / 2}$,

where $T_{e}$ is the electron temperature. In this work, we assumed isothermal electron with $T_{e}=T_{n}$, but is not necessarily a good assumption (Oyama et al., 1980). $T_{e}$, basically, should be equal to $T_{n}$, however, the few reports of electron temperature observations show that very often $T_{e}$ is higher than $T_{n}$ (Oyama, 2000, and references therein). For example, in the equatorial electrojet region, electron temperature measurement were only once reported by Sampath et al. (1974) and showed that $T_{e}$ would be approximately twice the $T_{n}$ obtained by models. Based on that, the isothermal electron assumption may lead to an underestimation of the vertical electric field by $\sim 8 \%$.

The gyrofrequencies were calculated, as given by:

$\Omega_{e, i}=\frac{q_{e, i} \cdot B}{m_{e, i}}$,

where $m_{e}$ is the electron mass and $m_{i}$ is the ion mass average, and $q_{e, i}$ is the electrical charge. The parameters used to perform the calculations of $\psi$ were obtained from Banks and Kockarts (1973), IRI Model (Bilitza, 2001), and IGRF Model (MacMillan and Maus, 2005).

In order to include the effect of vertical current in the magnetic equator, we used integration of the EEJ electric current equation along the magnetic field lines. Richmond (1973) stated that since the vertical electric field $\left(E_{z}\right)$ that drives the EEJ current is mapped along the magnetic field lines, its value should also depend on the conductivity integrated along the magnetic field line. For this reason we have chosen the ionospheric conductivity model developed by Denardini (2007), which calculate the Hall $\left(\sigma_{H}\right)$ and Pedersen $\left(\sigma_{P}\right)$ conductivities at each point of the geomagnetic field lines along the magnetic meridian at the radar site with height resolution of $1 \mathrm{~km}$, and then integrate them along the geomagnetic field line. Hence, the relation between the equatorial electric fields is given by:

$E_{o}=\frac{\int_{-\theta}^{+\theta} \sigma_{P} \cdot \boldsymbol{r} \cdot \boldsymbol{d} \boldsymbol{\theta}}{\int_{-\theta}^{+\theta} \sigma_{H} \cdot \boldsymbol{r} \cdot \boldsymbol{d} \boldsymbol{\theta}} \cdot E_{z} \Rightarrow E_{o}=\frac{\Sigma_{P}}{\Sigma_{H}} \cdot E_{z}$.

where $E_{o}$ is the E-region background electric field, $\boldsymbol{r}$ is the position of the magnetic field line element considering a dipole geometry, $\theta$ is the magnetic latitude, $\boldsymbol{d} \boldsymbol{\theta}$ is the differential magnetic latitude element vector and the quantities $\Sigma_{H}$ and $\Sigma_{P}$ are the Hall and Pedersen field-line integrated conductivities, respectively.

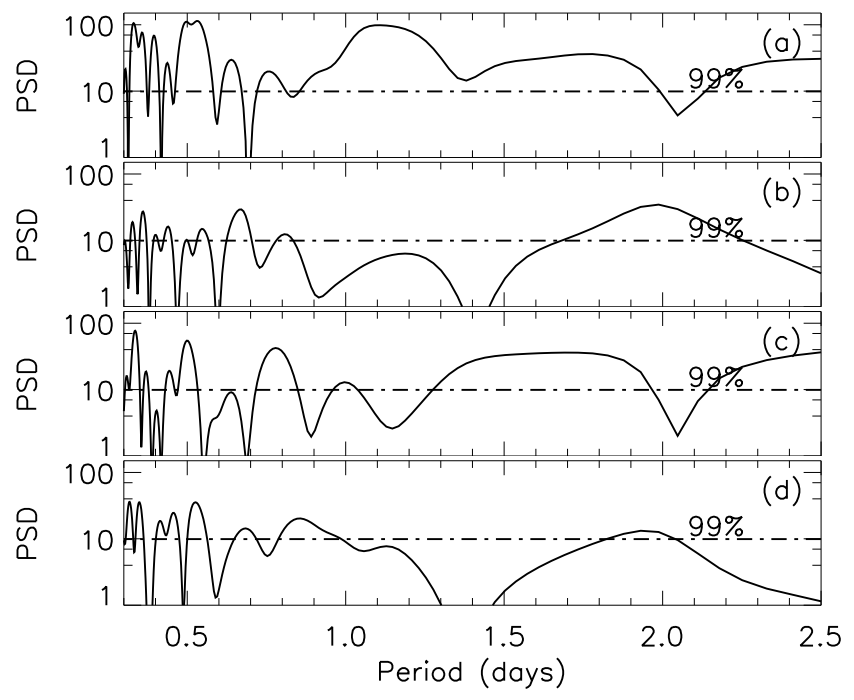

Fig. 1. Lomb-Scargle periodogram calculated for the zonal electric field for (a) 20-24 January, (b) 27-31 January, (c) 17-21 February, and (d) 24-28 February, in 2003. The horizontal (dash dot) line indicates the level of $99 \%$ of confidence $\left(=9.90 \mathrm{mV}^{2} / \mathrm{m}^{2}\right)$.

\subsection{Results}

In order to obtain information on the periodicity of the background E-region zonal electric fields, we performed a spectral analysis. We analyze 10 days in January (20-24 and 27$31)$ and 10 days in February (17-21 and 24-28). The LombScargle algorithm, which is used for extracting frequency components of unevenly sampled signals (Lomb, 1976; Scargle, 1982) and for series with gaps, has been used. In this case, the reason for using this method instead of a simpler Fourier Transform is that the RESCO radar has operated only between 08:00-18:00 LT, subsequently leaving gaps in the time series. Figure 1 shows the Lomb-Scargle periodogram calculated for the vertical electric fields for (a) 20-24 January, (b) 27-31 January, (c) 17-21 February, and (d) 24-28 February, in 2003. The horizontal (dash dot) line indicates the level of $99 \%$ of confidence $\left(=9.90 \mathrm{mV}^{2} / \mathrm{m}^{2}\right)$. In general, the periodograms show two remarkable period peaks below 1-day: $\sim 0.5$ - and 1.0-day. It indicates an electric field arising from the semidiurnal and diurnal tidal winds, respectively. Signatures of (exactly) 2-day waves are seen in the period of 27-31 January, with an anticorrelation between this wave and the tidal modes. Also, in February, the electric fields show remarkable periodicites of 1.9 days (Fig. 1d). Although, they do not show the same anticorrelation observed between the 2-day wave and the tidal modes. 
Table 1. Geographic coordinates and magnetic latitude of the magnetometer stations.

\begin{tabular}{crrr}
\hline Station Code & Mag. Lat. & Latitude & Longitude \\
\hline ASC & -2.36 & -7.95 & -14.38 \\
SLZ & -2.25 & -2.58 & -44.23 \\
HUA & -1.80 & -12.04 & -75.32 \\
TIR & -0.17 & 8.70 & 77.80 \\
PND & 2.85 & 11.92 & 79.92 \\
BNG & 4.20 & 4.33 & 18.57 \\
GUA & 5.30 & 13.59 & 144.87 \\
AAE & 5.32 & 9.03 & 38.77 \\
\hline
\end{tabular}

\section{Two-day signature in the EEJ currents}

\subsection{Magnetometer data}

Hourly geomagnetic data were obtained for 8 stations close to the magnetic equator (magnetic lat. $<\sim 5^{\circ}$ ). The data set were provided in HDZ or XYZ format. Data in XYZ were converted to HDZ format. We have calculated the Hcomponent variation with respect to the midnight base level, denominated as $d H . d H$ observed at a point on the ground is not only due to the overhead currents, but also due to the currents flowing over a latitudinal extent of a few degrees on either side of the overhead point (Vikramkumar et al., 1987). Also, the observed $d H$ variations at ground levels consists of two components: one due to currents in the EEJ itself $\left(d H_{\text {ext }}\right)$, and other due to currents induced by the electrojet in the earth $\left(d H_{\text {int }}\right)$. Since latitudinal distribution of the heightintegrated current intensity in the EEJ $(J)$ shows a maximum in magnetic equator and a sharp decay off-equator, and assuming a ribbon-like current flow, the ground level perturbations $d H_{\text {ext }}$ is related to the EEJ current by (Vikramkumar et al., 1987):

$d H_{\mathrm{ext}}=\frac{h_{0} \mu_{0}}{2 \pi} \int_{-10}^{+10} \frac{J(\lambda) d \lambda}{h_{0}^{2}+x^{2}(\lambda)}$,

where $h_{0}$ is the mean electrojet height, $x$ is the distance of the latitudinal element $d \lambda$ from the geomagnetic equator and $\mu_{0}$ is the permittivity of free-space. $J$ is integrated between $\pm 10^{\circ}$ geomagnetic latitude because the current contribution to $J$ is almost negligible beyond this range (Vikramkumar et al., 1987). Table 1 lists the geographic coordinates and magnetic latitude of the magnetometer stations.

External current systems, the equatorial electrojet in this case, induce secondary currents in the Earth; and their magnetic field is present at the Earth's surface as internal contributions (Olsen, 1997). Since the latter is the effect and the former the cause, the corresponding geoelectric currents are well correlated with the magnetic variations of ionospheric origin. Many studies related to $H_{\text {int }} / H_{\text {ext }}$ have estimated that the ratio vary between $0.39-0.70$ for different longitude zones (Vikramkumar et al., 1987, and references therein). Sampath and Sastry (1979), based on their rocket-borne observations of the electrojet current near Thumba, showed that the induced current is only $39 \%$ of the jet current. This is almost equal to the globally averaged value of $40 \%$ obtained by Duhau and Osella (1982). Also, Vikramkumar et al. (1987) apply an approximation of $\Delta \mathrm{H}_{\mathrm{ext}}=0.72 \times \Delta \mathrm{H}$ to compare observed $\Delta \mathrm{H}$ values with those estimated from measured electric fields. Based on that, the $d H \approx d H_{\text {ext }}$ assumption (neglecting the internal effects) may lead to an overestimation of the magnetic induction by EEJ currents. Still, we have restricted our analysis to magnetometer stations close to the magnetic equator (magnetic lat. $<\sim 5^{\circ}$ ), so $d H$ is supposed to contain mainly the EEJ influence. Another sources that may have some influence in $d H$ data (e.g., from magnetospheric sources) are discussed in Sect. 5.

\subsection{Wavelet analysis}

The wave-type oscillations were analyzed through spectral analysis of the magnetometer data using the wavelet transform. The wavelet-mother that best fitted to our objectives was Morlet, which is a plane wave modulated by a Gaussian envelope of unit width (Farge, 1992). The continuous wavelet transform (CWT) of a discrete sequence $x_{n}$ is defined as the convolution of $x_{n}$ with a scaled and translated version of the wavelet-mother or, by the convolution theorem, it is the inverse Fourier transform of the product in the frequency domain, as given by (Torrence and Compo, 1998):

$W_{n}(s)=\left(\frac{2 \pi s}{\delta t}\right) \cdot \sum_{k=0}^{N-1} \hat{x}_{k} \hat{\psi}_{0}^{*}\left(s \omega_{k}\right) \exp \left(i \omega_{k} n \delta t\right)$,

where $s$ is the wavelet scale, $k$ is the frequency index, $\delta t$ is the time resolution, $N$ is the total number of points, $\hat{x}$ is the Fourier transform of the time series, $\hat{\psi}_{0}^{*}\left(s \omega_{k}\right)$ is the complex conjugate of the wavelet-mother, and the angular frequency is defined as

$\omega_{k}=\left\{\begin{array}{l}+\frac{2 \pi k}{N \delta t}: k \leq N / 2 \\ -\frac{2 \pi k}{N \delta t}: k>N / 2\end{array}\right.$.

The Morlet wavelet-mother is defined as (Torrence and Compo, 1998):

$\hat{\psi}_{0}\left(s \omega_{k}\right)=\pi^{-1 / 4} H(\omega) \exp \left[-\frac{\left(s \omega-\omega_{0}\right)^{2}}{2}\right]$,

where $H(\omega)$ is the Heaviside step function $(H(\omega)=1$ if $\omega>0$, $H(\omega)=0$ otherwise) and $\omega_{0}$ is the frequency. The total energy is conserved under the wavelet transform, and the spacescale energy density for 1-D time series is defined as (Farge, 1992):

$E_{n}(s)=\frac{\left|W_{n}(s)\right|^{2}}{s}$. 


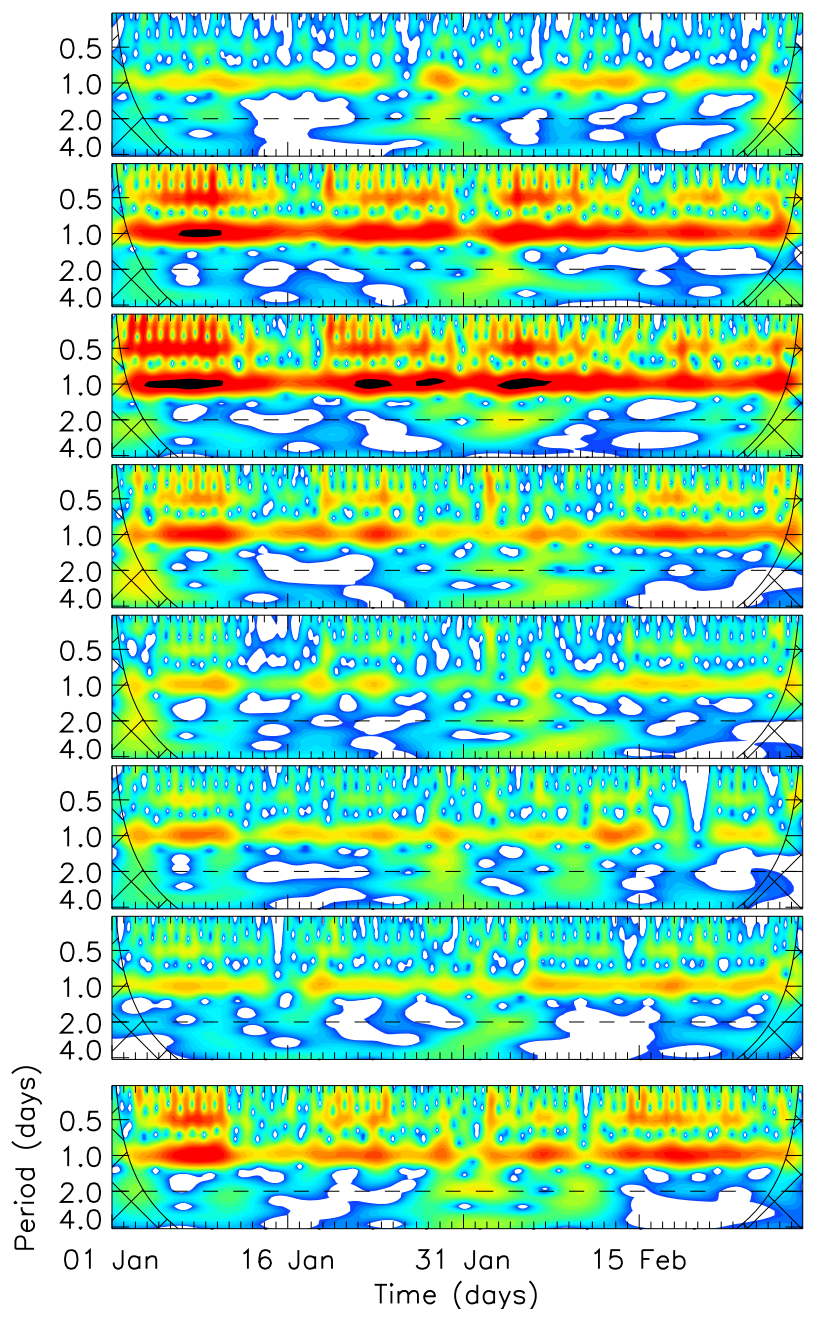

\begin{tabular}{lllllll}
\hline & & & & & & \\
2.0 & 2.6 & 3.2 & 3.8 & 4.4 & 5.0
\end{tabular}

Fig. 2. Space-scale energy density in log-scale of dH in JanuaryFebruary 2003 for the eight magnetic stations (from top to bottom): ASC, SLZ, HUA, TIR, PND, BNG, GUA, and AAE.

\subsection{Results}

Figure 2 shows the wavelet analysis of $d H$, performed using $1 \mathrm{~h}$ of time resolution, and 0.125 of scale resolution, to the 8 selected magnetic stations for periodicities in the range 0.25-4.0-days in January-February 2003. The overall results show that the diurnal and semidiurnal tides are higher than the 2-day wave modulation in $d H$ almost all the time. Stations close to dip equator show strong tidal modulation in $d H$. The only exception is the AAE station which is located in $5.32^{\circ}$ of magnetic latitude. Two-day wave modulation of $d H$ is observed in all stations: ASC for 26-31 January, SLZ for 1-8 February, HUA for 1-10 February, TIR for 30
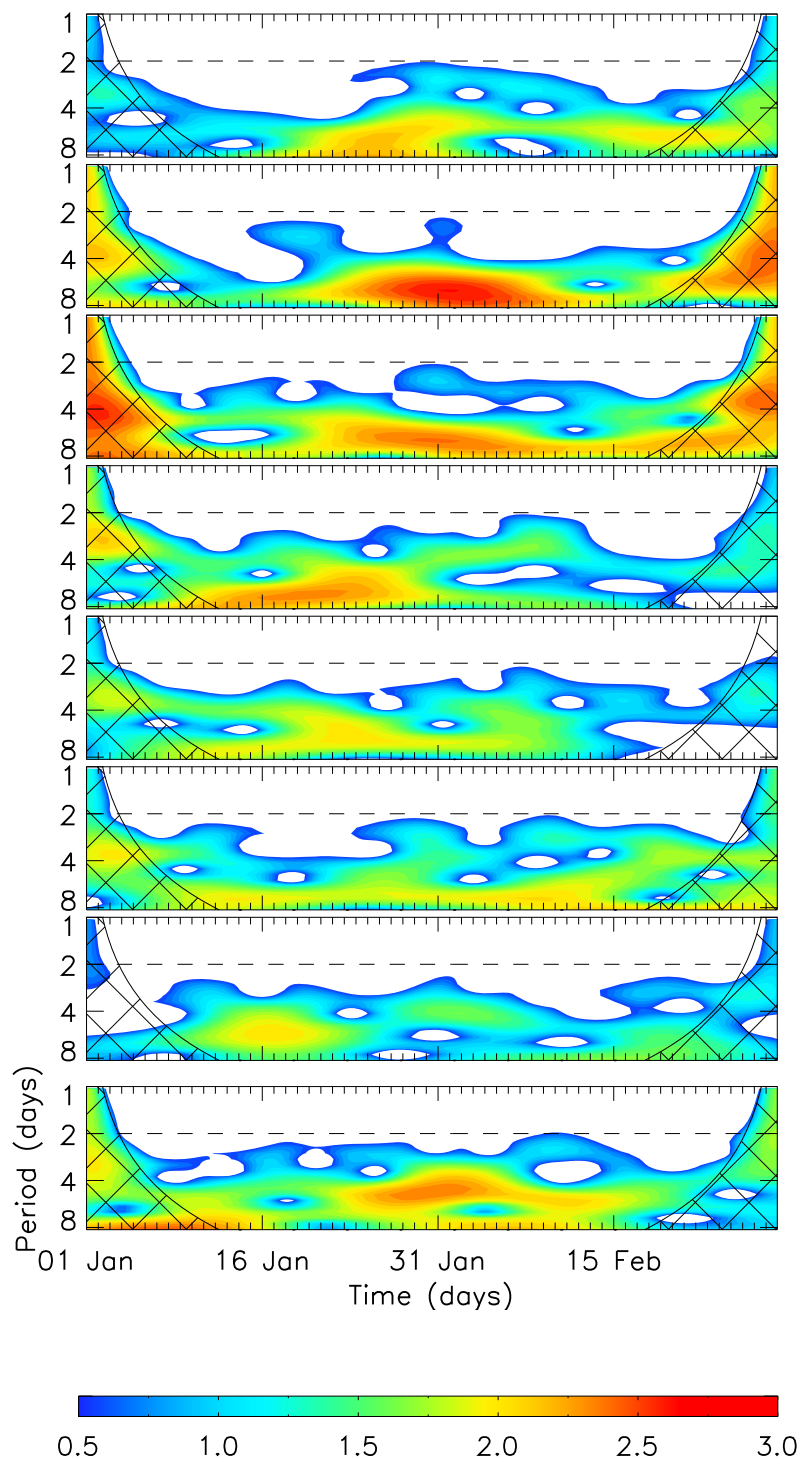

Fig. 3. Space-scale energy density in log-scale of the amplitude of the diurnal tide (0.90-1.15 days) in January-February 2003 for the eight magnetic stations (from top to bottom): ASC, SLZ, HUA, TIR, PND, BNG, GUA, and AAE.

January-7 February, PND for 30 January-10 February, BNG for 27 January-1 February, GUA for 31 January-8 February, and AAE for 27 January-13 February. Despite being the most remarkable periods with 2-day modulation, there also are others like during 18-23 February in HUA.

Figure 3 presents the wavelet analysis of the amplitude of the diurnal tide in $d H$ for the same magnetic stations and period as in Fig. 2. Two-day modulation of diurnal tide is not clearly observed. Actually the dominant periodicities are in the range from 4-8 days. We still observed some occurrences of quasi-2-day periodicities after 28 January for stations closer to dip equator. Figure 4 presents the wavelet 

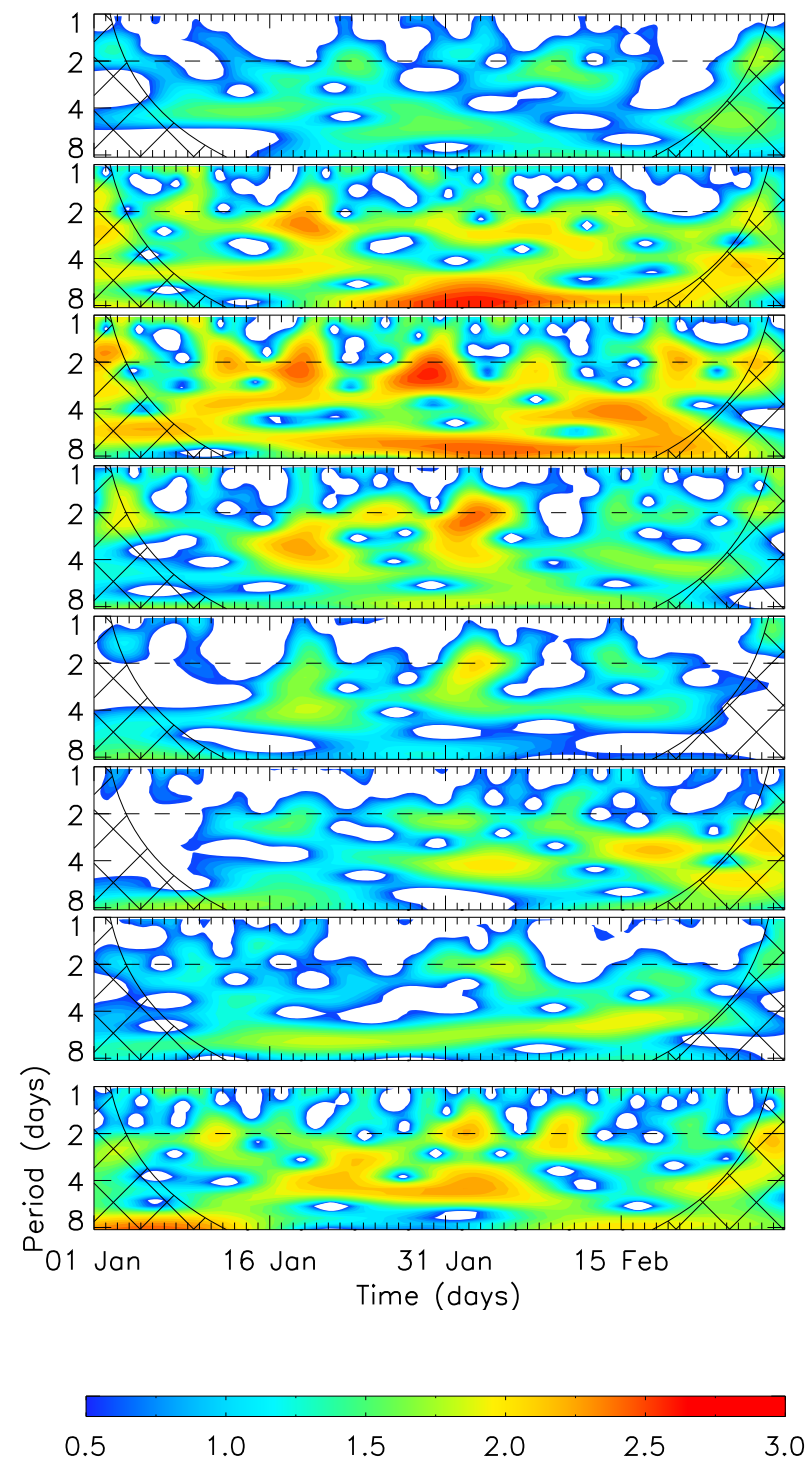

Fig. 4. Space-scale energy density in log-scale of the amplitude of the semidiurnal tide (0.40-0.60 days) in January-February 2003 for the eight magnetic stations (from top to bottom): ASC, SLZ, HUA, TIR, PND, BNG, GUA, and AAE.

analysis of the amplitude of the semidiurnal tide in $d H$. Twoday modulation of semidiurnal tide is clearly observed for almost all magnetometer stations, although there are other remarkable periodicities. The 2-day modulation of the semidiurnal tide seems to appear between 10 January-10 February. The events are not continous in all the period, they appear as burst-like occurrences with 5-10 days persistence. Another interesting feature is the fact that sometimes the 2-day wave signature is seen in the semidiurnal mode of $d H$, but it is not observed in the amplitude of $d H$.

\section{Previous reports of 2-day waves observations in January-February 2003}

Pancheva et al. (2006) analyzed the 2-day wave coupling of the low-latitude atmosphere-ionosphere system in the neutral winds by radar measurements located at four tropical stations, in the ionospheric electron density by seven ionosonde stations, and in the ionospheric electric currents by twentythree magnetometer stations, during the period between 1 December 2002 and 28 February 2003. The results of Pancheva et al. (2006) are summarized as follow:

- a burst-like 2-day wave activity prevailed in the tropical mesosphere/lower thermosphere (MLT) region having the following features: (a) the 2-day wave appeared in late December (around 25 December), peaked at most of the stations between 10 and 25 January, and persisted until early February; (b) westward propagation of the 2-day wave, but with no single wave number found; (c) vertical wavelengths were quite similar for all stations, $\sim 50$ to $65 \mathrm{~km}$; and (d) the 2-day wave appeared to penetrate into the dynamo region for part of the time (predominantly after 20 January); and

- simultaneous magnetometer data from a large number of stations in the tropical zone revealed the 2-day variability in the geomagnetic components appeared as modulations of the quiet-day Sq diurnal cycle, and ionosonde data from seven tropical stations indicated the same periodicy in $f o \mathrm{~F} 2$.

The 2-day modulation of the 24-h tidal amplitude at the initial and final stages of the 2-day wave activity is probably due to the observed anti-correlation between the 2-day wave activity and the diurnal tide (Pancheva et al., 2006, and references therein). They concluded that the main forcing agent in this atmosphere-ionosphere coupling seems to be the modulated tides, particularly the semidiurnal tide. The parameter that appears to be affected, and thus drives the observed 2day wave response of the ionosphere, is the dynamo electric field (Pancheva et al., 2006). They still suggest that 2-day wave response in the geomagnetic components does not appear to require the direct involvement of the 2-day wave in the vertical coupling (or at least not during the entire period).

\section{Discussions}

We have analyzed the indirect evidence of the presence of 2-day planetary wave oscillations in the EEJ using one VHF coherent radar and eight magnetometer stations located close to the dip equator. Our results are summarized as follow:

1. E-region background zonal electric field: (a) $E_{o}$ show a remarkable 2-day signature in the period 27-31 January; and (b) a anticorrelation between 2-day activity and the tidal modes (0.5-, and 1.0-days) is observed in the same period. 


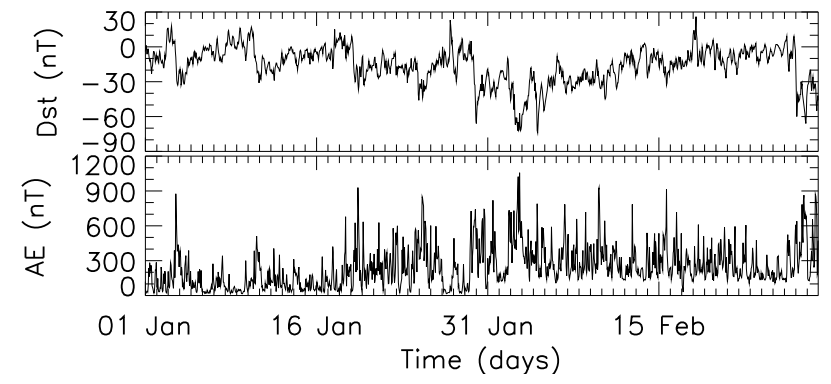

Fig. 5. $D_{s t}$ and AE indices for January-February 2003.

2. equatorial electrojet current: (a) the EEJ currents are clearly affected by the 2-day planetary wave; (b) 24-h tidal amplitude in ionospheric currents are not modulated by the 2-day wave. Actually, the tide that is modulated is the 12-h tide, i.e., the semidiurnal mode; and (c) the detection of the 2-day wave is better observed in the wavelet analysis of the semidiurnal tidal amplitude, rather than in the diurnal tidal amplitude and the $\mathrm{H}$-component variations.

The zonal electric fields inference has a dependence with the collision frequencies. According to Pancheva et al. (2006), the planetary waves may affect the collision frequencies through composition changes in the turbopause, but this effect is not so important as the dynamo electric field. Still, the zonal current density in the equatorial electrojet $\left(J_{o}\right.$, which mainly cause the $d H$ variation) is related to the $E_{o}$ through

$J_{o}=\left(\sigma_{P}+\frac{\sigma_{H}^{2}}{\sigma_{P}}\right) \cdot E_{o}$.

So, our results show good agreement between the observations of currents and electric fields. Also, despite of the fact that we have related the tidal wind systems as the main source of electric fields, two other physical processes can influence it:

- disturbance dynamo (Blanc and Richmond, 1980) - a current system driven by winds generated by external sources usually attributed to particle heating and Joule heating in the auroral zone; and

- penetration of high-latitude electric fields to low latitudes (Spiro et al., 1988) - the high-latitude ionospheric convection electric is not completely shielded from low and middle latitudes due to abnormal drift times of particles in the ring current.

Some short-term disturbances in the equatorial ionosphere may be caused by solar ionizing flux variations, and geomagnetic storms. Parish et al. (1994), for instance, point out a 13.5- and a 27-day oscillation in the EUV fluxes, which affect the $\Delta H$ variations through their influence on

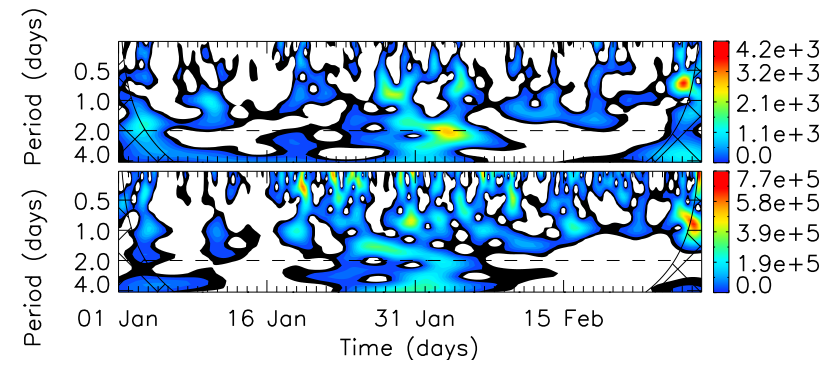

Fig. 6. Space-scale energy density of the $D_{s t}$ and AE indices for January-February 2003.

the E-region conductivity. We have analysed the geomagnetic activity based on the $D_{s t}$ and $\mathrm{AE}$ indices in order to distinguish peridiocities caused by magnetic storms and substorms, and those caused by "meteorological" sources. The $D_{s t}$ provide a good indication of the magnetic disturbance over low latitude, while the auroral indices in general would represent the degree of variability in the magnetospheric source of the disturbance electric field that penetrate to the equatorial latitudes. Figure 5 shows the magnetic indices and Fig. 6 presents the space-scale energy density of the indices. It can be seen that there are several small storms $\left(-50 \mathrm{nT} \leq D_{s t} \leq-30 \mathrm{nT}\right)$ and two moderate storms $\left(-100 \mathrm{nT} \leq D_{s t} \leq-50 \mathrm{nT}\right)$ in the periods $1-6$ and 26-28 February. The space-scale energy density points out a remarkable period with 2-day modulation of the indices from 1-6 February. It means that the 2-day signatures in the zonal electric fields and ionospheric currents observed in late January were possibly caused by ionospheric dynamo-planetary waves interaction, and not by magnetospheric disturbances. The results of Pancheva et al. (2006) support our analysis in the same way, since a 2-day wave activity is observed in the tropical MLT region with a remarkable 2-day modulation of the diurnal and semidiurnal tidal amplitudes at 27-31 January (simultaneously with our results).

\section{Conclusions}

This work intends to be the first attempting to demonstrate the signature of 2-day planetary wave in the dynamo electric field, based on E-region zonal electric fields inferred by a VHF coherent radar. A good agreement between our observations in the electric fields and ionospheric currents, and the meridional winds was found. An anticorrelation between the tidal periodicites (diurnal and semidiurnal) and the 2-day signature is shown in the electric fields. The 24-h tidal amplitude in ionospheric currents seem to be not modulated by the 2-day wave. The detection of the 2-day wave is better observed in the wavelet analysis of the semidiurnal tidal amplitude, rather than in the diurnal tidal amplitude and the $\mathrm{H}$ component variations. Finally, we point out that additional 
coordinated campaigns should be organized aiming further informations of the neutral atmosphere-ionosphere interactions.

Acknowledgements. The authors thank the World Data Center for Geomagnetism - Kyoto for data access and availability, and PDSC Almeida and LCA Resende for their help in radar data processing. One of the authors, HCA, thanks to CNPq/MCT for financial support to his MSc program through the Project nr. 131326/2007-4.

Topical Editor K. Kauristie thanks two anonymous referees for their help in evaluating this paper.

\section{References}

Abdu, M. A., Denardini, C. M., Sobral, J. H. A., Batista, I. S., Muralikrishna, P., Iyer, K. N., Veliz, O., and de Paula, E. R.: Equatorial electrojet $3 \mathrm{~m}$ irregularity dynamics during magnetic disturbances over Brazil: results from the new VHF radar at São Luís, J. Atmos. Sol.-Terr. Phys., 65(14-15), 1293-1308, 2003.

Abdu, M. A., Ramkumar, T. K., Batista, I. S., Brum, C. G. M., Takahashi, H., Reinisch, B. W., and Sobral, J. H. A.: Planetary wave signatures in the equatorial atmosphere-ionosphere system, and mesosphere-E- and F-region coupling, J. Atmos. Sol.-Terr. Phys., 68(3-5), 509-522, 2006.

Banks, P. M. and Kockarts, G.: Aeronomy (Part A), London: Academic Press, 1973.

Blanc, M. and Richmond, A. D.: The ionospheric disturbance dynamo, J. Geophys. Res., 85(A4), 1669-1686, 1980.

Bilitza, D.: International Reference Ionosphere 2000, Radio Sci., 36(2), 261-275, 2001.

Denardini. C. M.: A Conductivity Model for The Brazilian Equatorial E-Region: Initial Results, Braz. J. Geophys., 25(2), 87-94, 2007.

Duhau, S. and Osella, A. M.: A correlation between measured Eregion current and geomagnetic daily variation at equatorial latitudes, J. Geomag. Geoelectr., 34, 213-224, 1982.

Farge, M.: Wavelet Transforms and Their Applications to Turbulence, Annu. Rev. Fluid Mech., 24(3), 395-457, 1992.

Fejer, B. G. and Kelley, M. C.: Ionospheric Irregularities, Rev. Geophys. Space. Ge., 18(2), 401-454, 1980.

Forbes. J. M.: The equatorial electrojet, Rev. Geophys. Space. Ge., 19(3), 469-504, 1981.

Forbes, J. M.: Planetary waves in the thermosphere-ionosphere system, J. Geomagn. Geoelectr., 48, 91-98, 1996.

Forbes, J. M. and Leveroni, S.: Quasi 16-day oscillations in the ionosphere, Geophys. Res. Lett., 19, 981-984, 1992.

Forbes. J. M.: Tidal and Planetary Waves, in: The Upper Mesosphere and Lower Thermosphere: A Review of Experiment and Theory, edited by: Johnson, R. M. and Killeen, T. L., Geophysical Monograph Series, 87, Washington D.C., American Geophysical Union, 67-87, 1995.

Gurubaran, S., Ramkumar, T. K., Sridharan, S., and Rajaran, R.: Signatures of quasi-2-day planetary waves in the equatorial electrojet: results from simultaneous observations of mesospheric winds and geomagnetic field variations at low latitudes, J. Atmos. Sol. Terr. Phys., 63, 813-821, 2001.

Hagan, M. E., Forbes, J. M., and Vial, F.: Numerical investigation of the propagation of the quasi-two-day wave into the lower thermosphere, J. Geophys. Res., 98(D12), 23193-23205, 1993.
Kelley, M. C.: The Earth's Ionosphere - Plasma physics and electrodynamics, San Diego CA: Academic Press, 1989.

Lastovicka, J.: Forcing of the ionosphere by waves from below, J. Atmos. Sol.-Terr. Phys., 68, 479-497, 2006.

Lomb, N. R.: Least-squares frequency analysis of unequally spaced data, Astrophys. Space Sci., 39, 447-462, 1976.

Macmillan, S. and Maus, S.: International Geomagnetic Reference Field: The tenth generation, Earth Planets Space, 57(12), 11351140, 2005.

Olsen, N.: Geomagnetic tides and related phenomena, in: Tidal Phenomena, edited by: Wilhelm, H., Zurn, W., and Wenzel, H.G., 261-274, 1997.

Oyama, K. I., Hirao, K., Banks, P. M., and Williamson, P. R.: Is Te equal to $\mathrm{Tn}$ at the heights of 100 to $120 \mathrm{~km}$ ?, Planet. Space Sci., 28(3), 207-211, 1980.

Oyama, K. I.: Insitu measurements of Te in the lower ionosphere A Review, Adv. Space Res., 26(8), 1231-1240, 2000.

Pancheva, D. V., Mukhtarov, P. J., Shepherd, M. G., Mitchell, N. J., Fritts, D. C., Riggin, D. M., Franke, S. J., Batista, P. P., Abdu, M. A., Batista, I. S., Clemesha, B. R., and Kikuchi, T.: Two-day wave coupling of the low-latitude atmosphere-ionosphere system, J. Geophys. Res., 111, A7313, doi:10.1029/2005JA011562, 2006.

Pancheva, D. V., Mukhtarov, P. J., Mitchell, N. J., Fritts, D. C., Riggin, D. M., Takahashi, H., Batista, P. P., Clemesha, B. R., Gurubaran, S., and Ramkumar, G.: Planetary wave coupling (56-day waves) in the low latitude atmosphere-ionosphere system, J. Atmos. Sol.-Terr. Phys., 70, 101-122, 2008.

Reddy, C. A., Vikramkumar, B. T., and Viswanathan, K. S.: Electric Fields and currents in the equatorial electrojet deduced from VHF radar observations - I. A method of estimating electric fields, J. Atmos. Terr. Phy., 49(2), 183-191, 1987.

Parish, H. F., Forbes, J. M., and Kamalabadi, F.: Planetary waves and solar emission signals in the equatorial electrojet, J. Geophys. Res., 99, 355-368, 1994.

Richmond, A. D.: Equatorial Electrojet. 1. Development of a Model Including Winds and Instabilities, J. Atmos. Terr. Phys., 35(6), 1083-1103, 1973.

Sampath, S., Sastry, T. S. G., Oyama, K., and Hirao, K.: Joule heating due to the equatorial electrojet as observed by rocketborne probes, Space Res., 14, 253-258, 1974.

Sampath, S. and Sastry, T. S. G.: Results from in situ measurements of ionospheric currents in the equatorial region, J. Geomagn. Geoelectr., 31, 373-379, 1979.

Scargle, J. D.: Studies in astronomical time series analysis. II. Statistical aspects of spectral analysis of unevenly spaced data, Astrophys. J., 263, 835-853, 1982.

Spiro, R. W., Wolf, R. A., and Fejer, B. G.: Penetration of highlatitude electric field effects to low latitudes during SUNDIAL 1984, Ann. Geophys., 6, 39-50, 1988.

Torrence, C. and Compo, G.: A practical guide to wavelet analysis, B. Am. Meteorol. Soc., 79, 61-78, 1998.

Vikramkumar, B. T., Rao, P. B., Viswanathan, K. S., and Reddy, C. A.: Electric Fields and currents in the equatorial electrojet deduced from VHF radar observations - III. Comparison of observed $\Delta H$ values with those estimated from measured electric fields, J. Atmos. Terr. Phys., 49(2), 201-207, 1987. 